\title{
Node Degree Based Routing Metric for Traffic Load Distribution in the Internet*
}

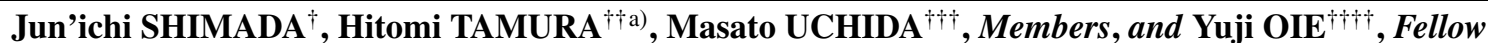

\begin{abstract}
SUMMARY Congestion inherently occurs on the Internet due to traffic concentration on certain nodes or links of networks. The traffic concentration is caused by inefficient use of topological information of networks in existing routing protocols, which reduces to inefficient mapping between traffic demands and network resources. Actually, the route with minimum cost, i.e., number of hops, selected as a transmission route by existing routing protocols would pass through specific nodes with common topological characteristics that could contribute to a large improvement in minimizing the cost. However, this would result in traffic concentration on such specific nodes. Therefore, we propose a measure of the distance between two nodes that is suitable for reducing traffic concentration on specific nodes. To consider the topological characteristics of the congestion points of networks, we define node-to-node distance by using a generalized norm, $\mathrm{p}$ norm, of a vector of which elements are degrees of intermediate nodes of the route. Simulation results show that both the maximum Stress Centrality (SC) and the coefficient of variation of the SC are minimized in some network topologies by selecting transmission routes based on the proposed measure of node-to-node distance.

key words: traffic load distribution, routing metric, node degree, p-norm, stress centrality
\end{abstract}

\section{Introduction}

Congestion inherently occurs on the Internet, because traffic concentrates on certain links or nodes. The traffic concentration is caused by inefficient mapping between traffic demands and network resources, because existing routing protocols are not aware of both traffic demands and the topological features of networks. Routing protocols consider the total communication cost of a route between two arbitrary communication nodes in networks as the distance between nodes. More precisely, the existing routing protocols apply the number of hops or an inverse number of the physical bandwidth of a route as the cost. Then, protocols select the route with the minimum cost as the transmission route, that is, the transmission route between two communication

\footnotetext{
Manuscript received June 5, 2012.

Manuscript revised October 1, 2012.

${ }^{\dagger}$ The author is with Japan Advanced Institute of Science and Technology, Nomi-shi, 923-1292 Japan.

${ }^{\dagger}$ The author is with the Department of Information Electronics, Faculty of Engineering, Fukuoka Institute of Technology, Fukuoka-shi, 811-0295 Japan.

${ }^{\dagger \dagger}$ The author is with the Department of Electrical, Electronics and Computer Engineering, Faculty of Engineering, Chiba Institute of Technology, Narashino-shi, 275-0016 Japan.

titt The author is with the Faculty of Computer Sciences \& Systems Engineering, Kyushu Institute of Technology, Iizuka-shi, 820-8502 Japan.

${ }^{*}$ A part of this paper was presented at HEUNET 2011.

a)E-mail: h-tamura@ fit.ac.jp

DOI: $10.1587 /$ transinf.E96.D.202
}

nodes has shortest distance between them. The minimum cost route does not change even if some nodes and links are in congestion.

Traffic Engineering (TE) [1] is one solution to address the congestion problem in the IP layer by adaptive routing. Many TE algorithms have been proposed, and all of them suggest dynamically change the transmission path depending on the resource utilization of the path [2]. TE algorithms are aware of both traffic fluctuations and resource utilization in a short period of time; however, existing TE schemes are not aware of the underlying network topology. These schemes rely on transmission delay or packet losses of a route between two arbitrary communication nodes in networks and define the distance between two communication nodes by the transmission delay or packet losses. Because these distance measures fluctuate over time, route oscillation may occur in the existing TE schemes.

To alleviate congestion on the Internet, the topological features of the network should be considered, because the number of alternative paths and the path diversity between two communication nodes highly depend on the network topology. Recent studies have demonstrated the scale-free property of the Internet, e.g., in Autonomous System (AS)level, application-level (i.e., World Wide Web), and routerlevel topologies [3]-[6]. The scale-free property is defined by a power-law distribution $P(k) \sim k^{-\gamma}$, where $k$ represents the number of links per node (called "degree" or "node degree") in terms of node connectivity. The power-law property of network topology means that a network consists of a small number nodes having high connectivity and a large number of nodes having relatively low connectivity. In such networks, the number of hops between two arbitrary communication nodes is relatively small even if many nodes are present in the network, because nodes with a significant high node degree can make the so-called small-world property. In addition, some studies showed that the power-law distribution of node connectivity provides robustness of connectivity and fault tolerance in large-scale wireless networks, e.g., Mobile Ad-hoc Networks (MANETs) and Wireless Sensor Networks (WSNs) [7], because scale-free networks are highly resilient to random errors and attacks. However, scale-free networks are vulnerable to attacks and errors targeting a few high-degree nodes and may suffer from traffic concentration in them. Thus, we regard node degree as an important factor that roughly represents the relationship between network topological characteristics and traffic load.

Some related studies addressed the network congestion 
issue in the large-scale networks by using the node degree information in route decision [12], [13]. However, they evaluated only some characteristics of the routing metric in BA (Barabási-Albert) networks. In this paper, we employ $p$ norm of node degree for link metrics as an extension of the metrics we have proposed so far, and examine its characteristics in some networks including BA, and more practical topologies of ISP networks. Furthermore, we will show how the traffic load is well distributed by using appropriate $p$. In addition, we propose an implementation method of the proposed routing metric in existing networks, and evaluate the performance of the proposed scheme in some network topologies. Finally, we clarify the applicability of the proposed scheme in real Internet Service Provider (ISP) networks.

The rest of the paper is organized as follows. In Sect. 2, some existing studies related to topology estimation of the Internet and TE schemes are presented. In Sect. 3, we review our previous proposal of shortest path selection based on node degree and propose a new definition of distance by considering the sequencee of node degree from source to destination. In Sect. 4, we explain our simulation settings for the evaluation. Then, we show the basic performance of the proposed scheme and investigate the applicability of the proposed scheme for some real network topologies in Sect. 5. Finally, in Sect. 6, a brief conclusion is given.

\section{Related Works}

Recently, many studies have focused on the topological characteristics of some network levels of the Internet. Reference [3] first showed scale-free properties of an interdomain level topology by combining routing tables collected from many Border Gateway Protocol (BGP) routers. Furthermore, the Cooperative Association for Internet Data Analysis (CAIDA) carries out projects to analyze ASlevel [4] and router-level topologies [5]. In [4], properties of an AS-level topology were investigated. To obtain a probable AS-level topology, the following three data sources were integrated; traceroute, routing information extracted from the BGP, and the WHOIS database. The authors of that study demonstrated that the degree distribution of the topology followed a power-law. In another study, ISPlevel topologies were extracted by end-to-end measurement with traceroute and were shown to have a scale-free nature [8]. Additionally, many researchers have focused on the topology generation of realistic networks [9]-[11]. In [9], since some possible graph topologies are based on the same node degree distribution, a realistic router-level topology was inferred by considering the routers' technological constraints. This work was validated by examining the router-level topology of some real network topologies. The primary contribution of the papers discussed above is the clarification that the real network topology is characterized by the node degree distribution. In this paper, we focus on the node degree.

Next, routing metrics, namely, the distance between a node pair in the existing routing protocols, such as the Routing Information Protocol (RIP) and the Open Shortest Path First (OSPF) protocol, are defined by the number of intermediate routers (hops) or the total link cost between a source and its destination node. The link cost is usually defined as equal value, or the inverse number of the link bandwidth. Therefore, the transmission path is not changed as long as the network topology does not vary. In addition, some TE schemes use packet-loss ratio, utilization, and throughput of a link or a path as the routing metric. That is, the distance between a node pair is defined as the packet-loss ratio, utilization, and throughput of a path. Therefore, the transmission path can often change depending on traffic fluctuation. The traffic fluctuation can cause route oscillation, and the route oscillation can affect the packet transmission. As discussed above, the definition of the distance in networks does not address the topological characteristics of networks.

Some studies focusing on the traffic load distribution in large-scale networks proposed node degree based routing. In [12], a threshold-based scheme for distributing generated packets to several paths from the source to the destination was presented. When the number of packets generated in the network was more than the threshold, the transmission path of each packet was determined by the sum of the node degrees to the power of $p$ along the path. The authors of that study evaluated the critical value $\mathrm{Rc}$ (packets generated in the system within unit time) where a phase transition takes place from free flow to congested traffic, and they mainly analyzed its characteristics in BA model, and Rc in BA model for $p=0$ to 2 in [12]. Furthermore, under the assumption that high-degree nodes have stronger packet-processing ability, the next-hop node of each packet was determined in a probabilistic way based on the outgoing degree of the next-hop node and the path length from the source node to the destination node [13].

In [14], we proposed a shortest path selection scheme using the node degree among multiple shortest paths. That is, the proposed scheme defined the distance between two arbitrary nodes by both the number of hops and the node degree. The scheme selected the transmission path to best mitigate congestion in some parts of the network judged by the node degree, under the constraint that the selected transmission path has the same number of hops as the shortest path. However, our previous proposal was difficult to implement due to its computational complexity in finding all shortest paths between a node pair. Here, we employ the $p$-norm defined later as a routing metric, which is easy to implement in the existing networks.

\section{Transmission Route Decision by Node Degree Infor- mation for the Traffic Load Distribution}

In [14] and [18], we proposed route selection schemes for the traffic load distribution in large-scale network. These proposed schemes use node degree as a parameter. In [14], we compared the value of the node degree along the path (see Sect. 3.1 for details). In [18], we defined and calcu- 
lated the link $\operatorname{cost}(\mathrm{C})$ set by degrees(n) of two neighboring $\operatorname{nodes}(\mathrm{i}, \mathrm{j})$.

$$
C_{i j}=\frac{n_{i}^{p}+n_{j}^{p}}{n_{i}^{p-1}+n_{j}^{p-1}}
$$

This scheme selects the path that has the minimum sum of $C_{i} j$ (called the Lehmer means) from source to destination. These previous schemes tend to select a path determined by the magnitude of the degree on specific node. We have focused on the path between the source and destination, and executed mathematical analysis to evaluate the relations between the length of the path and the node degree along the path. Furthermore, we propose a generalized definition of the distance between two arbitrary nodes in large-scale networks by using both the node degree information and the definition of the $p$-norm. Then, we show how to use the new definition of the distance to select a transmission route between two arbitrary nodes.

\subsection{Route Selection Scheme Based on Node Degree among Multiple Shortest Paths}

In [14], we proposed a route selection scheme with the node degree among multiple shortest paths from a source to its destination in large-scale networks. The paper showed that large-scale networks have many shortest paths, namely, Equal Cost Multi-Paths (ECMP), between arbitrary node pairs compared to random networks. Therefore, in the proposed scheme, we assumed that a node knew all the shortest paths to its destination. We proposed two path selection schemes based on the node degree of the shortest paths; the Minimizing the Maximum node Degree along the path (MMD) scheme and the Minimizing the Sum of node Degree along the path (MSD) scheme.

As shown in Fig. 1, node $i$ has degree $k_{i}$, and some shortest paths are possible with $m$ hops between a source node $s$ and a destination node $d$. Here, we assume that the node degree is the number of outbound links of a node, that is, node $i$ with degree $k_{i}$ has $k_{i}$ outgoing links. In the MMD scheme, the maximum node degree of route $j$, which is $D_{\max (j)}=\max _{i \in V_{j}} k_{i}\left(V_{j}\right.$ : a set of nodes on the shortest path $j$ between nodes $s$ and $d$ ), is the measure for route selection, and then the source node selects a route having the minimum of the maximum node degree among the multiple shortest paths to its destination. Hence, the route with $\min _{j \in P_{s d}} D_{\max (j)}\left(P_{s d}\right.$ : the set of shortest paths between the source node $s$ and the destination node $d$ ) is selected. Thus, the MMD scheme tries to mitigate the impact of traffic concentration on relatively high-degree nodes by explicitly avoiding high-degree nodes.

In the MSD scheme, the sum of the node degrees, $S_{j}=\sum_{i \in V_{j}} k_{i}$, along a shortest path $j\left(j \in P_{s d}\right)$ is the measure for route selection, and then the source node selects a route having the minimum of the sum of node degrees along a shortest path. Therefore, the route with $\min _{j \in P_{s d}} S_{j}$ is selected. In the MSD scheme, a route tries to implicitly avoid

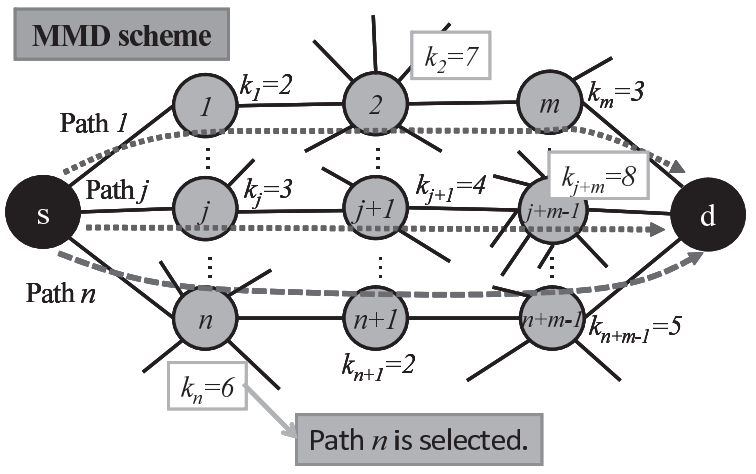

(a) MMD.

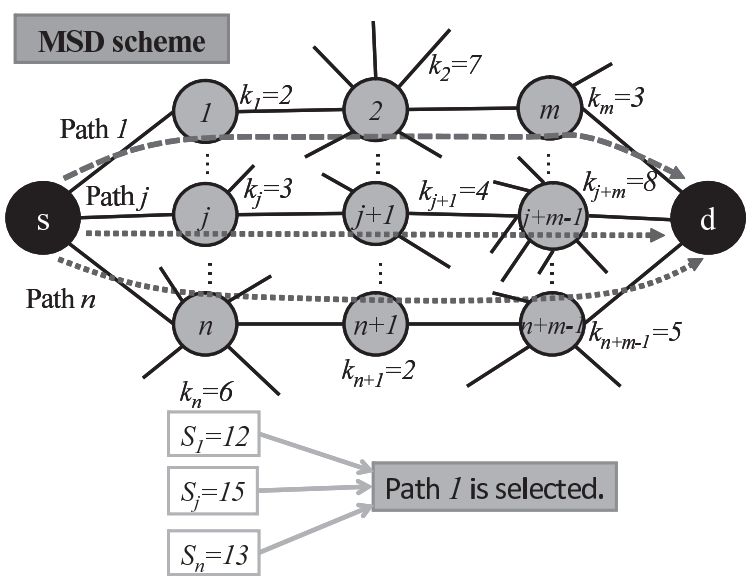

(b) MSD.

Fig. 1 Examples of path selection in the MMD and the MSD schemes [14].

passing through high-degree nodes.

In the two schemes, we saw that the MSD scheme is more effective in traffic load distribution at a high-degree node in BA networks. However, both the MSD and the MMD schemes decided the transmission route only among the shortest paths from a source to its destination. Consequently, we saw that the load-distribution may not be as effective in these schemes for some realistic ISP topologies. Furthermore, the route calculation algorithms in both the MSD and the MMD have significant computational complexity, i.e., $O\left(N^{2}\right)$ ( $N$ : number of nodes in the network), because they need to know all equal cost shortest paths between two arbitrary nodes. Therefore, the schemes are not practical.

Even though the schemes are not practical, the schemes consume minimum network resources (e.g., nodes and links) in each node pair. Therefore, the transmission performance (e.g., transmission delay) of the communication between each node pair is not affected by changing the shortest path from the normal shortest path to the shortest path based on the MMD or the MSD scheme.

3.2 Route Selection Scheme Based on the Node Degree and the Definition of the Norm

Both the MMD and the MSD schemes described in $3.1 \mathrm{im}-$ 


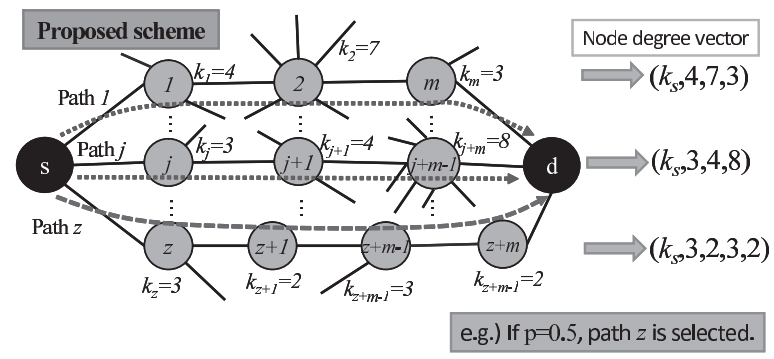

Fig. 2 An example of a node degree vector and a distance calculation using node degrees.

ply that the node degree, which is the number of outbound links of a node, is a good measure to represent the distance, including the congestion possibility, between a pair of nodes in large-scale networks. In this subsection, we provide a new definition of the node-to-node distance based on the node outgoing degree. Then, we also apply the definition of "norm" to calculate the distance between two arbitrary nodes. In addition, we discuss how to calculate a route between a node pair based on the norm-based distance in the existing routing protocol, and how to implement the route calculation.

\subsubsection{New Definition of Distance between Two Arbitrary Nodes (Mathematically Approach)}

A norm is the generalization of the length of a vector. For an $n$-dimensional vector, $\mathbf{x}=\left(x_{1}, x_{2}, \cdots, x_{n}\right)$, the $p$-norm is defined as

$$
\|x\|_{p}=\sqrt[p]{x_{1}^{p}+x_{2}^{p}+\cdots+x_{n}^{p}} \cdot(0<p)
$$

If $p=1$, the 1 -norm is equal to $\sum_{i=1}^{n} x_{i}$, while, $\infty$-norm is equal to $\max _{i} x_{i}$ when $p=\infty$. In addition, if the $p$-norm is used in path selection schemes which select one path among multiple shortest paths, the path selection scheme with $p=$ 1 becomes MSD, while that with $p=\infty$ becomes MMD.

Herein, we regard a sequence of node degree $k_{i}(i=$ $1,2, \cdots, n)$ of a path from a source to its destination as a vector (called the "node degree vector") as shown in Fig. 2. Then, we define a routing metric from a source to its destination as the $p$-norm of the node degree vector, and regard the route minimizing the $p$-norm as a forwarding route.

\subsubsection{Implementation of the Norm-Based Routing Metric in a Routing Protocol}

Here we suppose that each node's degree, which is defined as the number of outgoing links of a node, is propagated to other nodes in a network by the route control message of the routing protocol, e.g., OSPF. As shown in Fig. 3, the link cost, $C_{q r}$, of a link from node $q$ to node $r$ of an outbound link attached to the node $q$ having $k_{q}$ links is defined as $k_{q}$. Likewise, the link cost, $C_{r q}$, of a link from node $r$ to node $q$ of an outbound link attached to the node $r$ having $k_{r}$ links is defined as $k_{r}$. Then, a route having the minimum $p$-norm of

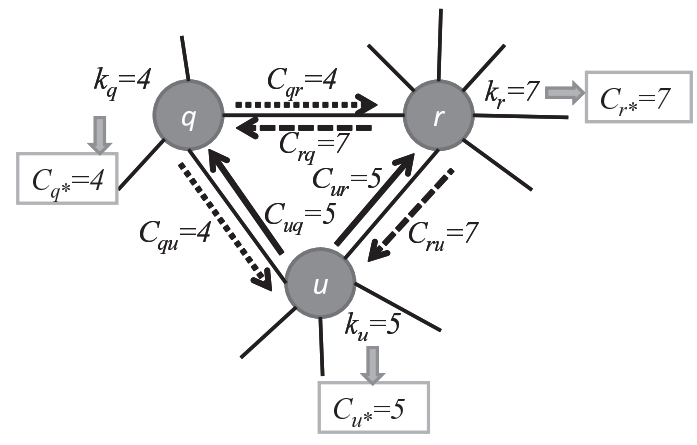

Fig. 3 An example of link cost settings.

the node degree vector is decided by a modified Dijkstra's algorithm.

The normal Dijkstra's algorithm calculates the sum of the costs from a source to its destination and selects a route minimizing the sum of these costs. In our scheme, $\|x\|_{p}=\sqrt[p]{x_{1}^{p}+x_{2}^{p}+\cdots+x_{n}^{p}}$ of the $p$-norm should be minimized, so we modify Dijkstra's algorithm to calculate the $p$-norm of the link costs along a route. Thus, our proposed routing metric is realized by a slight modification of the existing routing protocol. Therefore, the computational complexity of the route calculation algorithm is $O(M \log N)(M$ : the number of links in the network, $N$ : the number of nodes in the network), because the complexity of the proposed scheme is the same as that of Dijkstra's algorithm. Hence, the proposed scheme is practical in existing networks. Furthermore, the link costs are asymmetric in the opposite direction of a link, and so the transmission routes are expected to be distributed in the network. However, because the proposed scheme uses the node degree to measure the node-to-node distance, the transmission performance could be affected by the increment of the number of hops.

\section{Simulation Settings}

We investigate the performance of the proposed scheme by a computer simulation. To evaluate the basic characteristics of our proposed scheme, we first use the network topology generated by the BA model [15] with the number of nodes, 1000 , the number of links, 1997, and the lowest degree of a node, $m=2$. The power-law exponent of the node degree distribution is -2.77 in the network. By following the existing network configurations, we assume that the number of nodes is several hundred, the average degree is about $2 \sim 3$, and power-law exponent of the node degree distribution is about $-3 \sim-2$. Also, we assume that minimum of node degree is 2 in order to provide more than one route even in the source node. For comparison, we use the Waxman model as a random network topology model. In the random network, the number of nodes is set to 1000 , the number of links is 2000 , and the lowest degree of a node is set to 2 .

In addition, we use some router-level topologies in existing ISP networks to evaluate the applicability of the proposed scheme in a real network environment. The router- 
Table 1 Network topology models [17].

\begin{tabular}{|c|c|c|c|}
\hline $\begin{array}{c}\text { AS number } \\
\text { (ISP name) }\end{array}$ & 1239 (Sprint) & 3356 (Level3) & 7018 (AT\&T) \\
\hline \hline \# of nodes & 604 & 624 & 631 \\
\hline $\begin{array}{c}\text { Power-law } \\
\text { exponent }\end{array}$ & -3.18 & -2.54 & -3.45 \\
\hline \# of links & 2274 & 5300 & 2078 \\
\hline $\begin{array}{c}\text { max degree } \\
\text { of nodes }\end{array}$ & 44 & 169 & 43 \\
\hline av. of degree & 7.528 & 16.986 & 6.586 \\
\hline
\end{tabular}

level topologies are measured by Rocketfuel [8]. Table 1 summarizes the topological information of the real network topologies used in the evaluation.

If the common OSPF routing protocol is applied in the networks, we assume that the link cost of all links is equal. That is, the shortest path between a node pair has the minimum number of hops. Furthermore, if the MMD or the MSD scheme is applied, we also assume that the link costs are set equally for all links of the network.

A specific traffic model is not defined in this study. Therefore, we do not assume the link bandwidth of each link. We investigate performance of our proposal in a worstcase scenario, when all node pairs communicate at the same time. Therefore, we also do not consider the impact of the traffic matrix, i.e., the node pair of each communication and the time variation of the traffic amount. In this study, we focus on an appropriate mapping between the topology and the transmission route in the networks; for this reason, we have no interest in adaptive route selection in response to the traffic variation.

Instead, as a performance measure that represents the traffic load on each link, we use the Stress Centrality (SC) [16]. The Stress Centrality on link $l$ is defined as follows:

$$
S C(l)=\sum_{s \neq t \in V} \sigma_{s t}(l),
$$

where $s \in V$ and $t \in V$ represent nodes of the graph $G=(V, E)$, and $\sigma_{s t}(l)$ denotes the number of paths from source $s$ to destination $t$ on link $l$. If a path from $s$ to $t$ includes link $l$, then $\sigma_{s t}(l)=1$. Otherwise, $\sigma_{s t}(l)=0$. Thus, $S C(l)$ represents the maximum possible traffic load at link $l$ when all the node pairs simultaneously communicate in a network. We investigate the normalized $S C(l)$ in the evaluation section, because the ratio of $S C(l)$ to the total paths in the network indicates the probability of congestion level ranging from 0 to 1 . Note that, in contrast, the betweenness centrality, that is defined as $B C(i)=\sum_{s \neq t \in V} \delta_{s t}(i)(i \in V$ : node ID, $\delta_{s t}(i)$ : the number of paths from source $s$ to destination $t$ on node $i$ ), indicates the traffic load per node. We apply the SC as a measure of the traffic load per link. In the evaluation, we use the normalized $S C(l)$, which is divided by the total number of node pairs. If the normalized $S C(l)$ is equal to 1 , then all the paths between all the node pairs pass through link $l$. The objective is to minimize the maximum normalized $S C(l)$, and to minimize the coefficient of variance of normalized $S C(l)$.
We also investigate the number of hops of each route. In the proposed scheme, more than the shortest paths are considered as a transmission route from a source to its destination. Therefore, we investigate the number of hops as a rough representation of the transmission delay.

\section{Simulation Results and Discussion}

In this section, we show some simulation results. We focus on the impact of the new routing metric based on both the $p$ norm and the node degree on the SC of each link, the impact of parameter $p$ on the $\mathrm{SC}$, and the impact of $p$ on the number of hops of the transmission route.

\subsection{Basic Performance of the Common SPF Routing, the MMD and the MSD Schemes}

First, we briefly review the basic performance in the common Shortest Path First (SPF) routing, the MMD, and the MSD schemes in both BA and random networks. Figures 4, 5 , and 6 show the normalized $S C(l)$ on each node in each path selection scheme in the BA network, and Figs. 7, 8, and 9 show the normalized $S C(l)$ in the random network (Waxman model). The $\mathrm{x}$-axis indicates node degree, therefore, a point in the graph indicates the normalized Stress Centrality (SC) on a link attached to a node with degree $x$.

Next, we compare the performance of the three schemes in the BA network. As shown in Fig. 4, the normalized SC increases as the node degree increases; that is, the normalized SC is proportional to the node degree in the common SPF routing algorithm. In addition, in the common SPF routing, the variance of the normalized SC of each link is large.

In contrast, from Figs. 5 and 6, we can see that both the MMD and the MSD schemes can reduce the normalized SC in each node degree. In addition, the schemes can reduce the maximum normalized SC and reduce the variance of SC in comparison with the common SPF routing. In particular, the MSD scheme is more effective in reducing the variance of the normalized SC than is the MMD scheme. Since the MMD scheme explicitly avoids a path including the highestdegree node, it is more effective for reducing the SC at the high-degree nodes than is the common SPF routing. In addition, the MSD scheme selects the shortest path composed of low-degree nodes, so the normalized SC of high-degree nodes in the MSD scheme reduces more than those in the MMD scheme.

Last, we show the performance of the three schemes in the random network generated by the Waxman model. As shown in Fig. 7, the normalized SC is also proportional to the node degree in the common SPF routing algorithm in the random network. However, the maximum SC is lower than that in the BA network even if the number of nodes, the number of links, and the node pairs are almost the same. This is because the shortest paths are relatively spread out in the random network due to its random connectivity in the network. Hence, the random network has the possibility of 


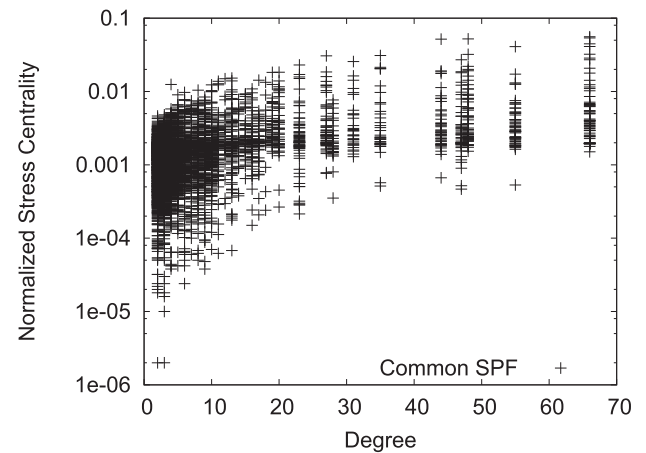

Fig. 4 Normalized SC of each link (Common SPF, BA model).

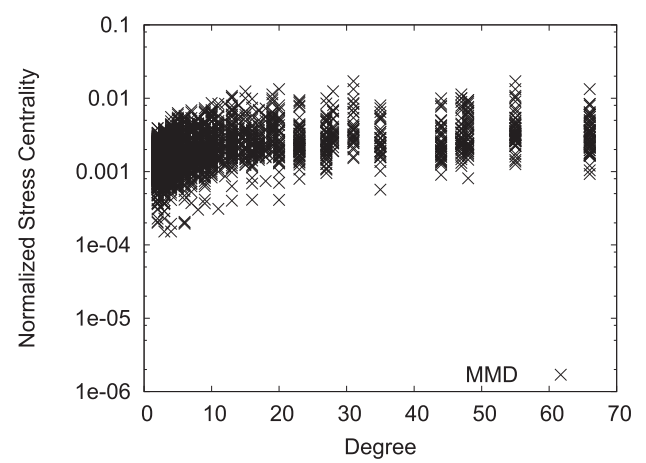

Fig. 5 Normalized SC of each link (MMD, BA model).

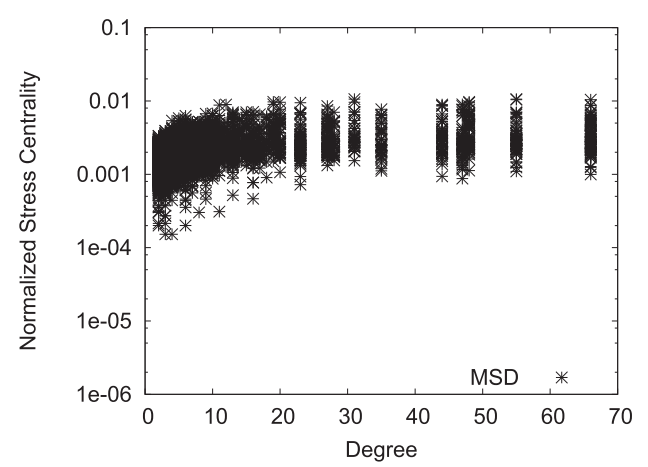

Fig. 6 Normalized SC of each link (MSD, BA model).

a traffic load distribution in its topological characteristics. However, if the MMD or the MSD scheme is applied in the random network, the normalized SC is also reduced in each link in the random network, as in the BA network. Therefore, we demonstrate that the shortest path selection, which is based on the degree of nodes along the shortest path, is effective for avoiding traffic concentration at a link attached to a high-degree node, independent of the network topological feature.

\subsection{Basic Performance of the Proposed Scheme}

Figures 10,11, and 12 show the normalized SC of each link when the $p$-norm of the node degree vector is applied to the routing metric in the BA network. In the figures, the $x$-axis

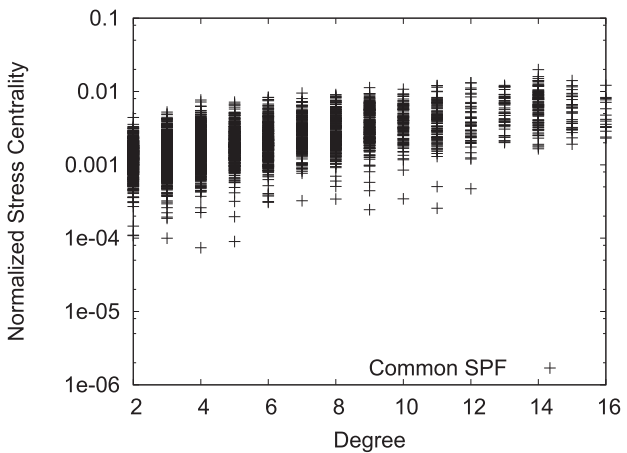

Fig. 7 Normalized SC of each link (Common SPF, Waxman model).

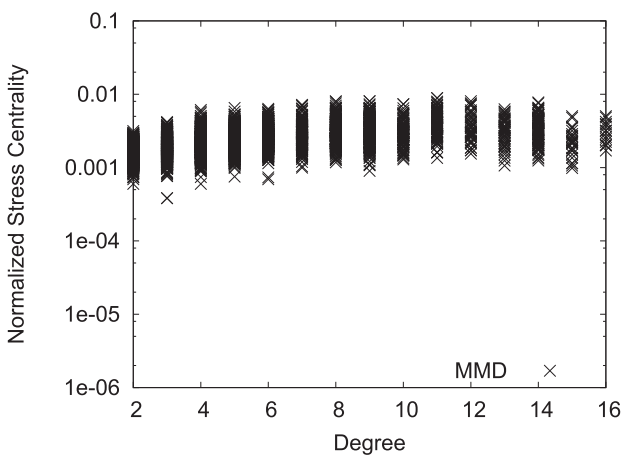

Fig. 8 Normalized SC of each link (MMD, Waxman model).

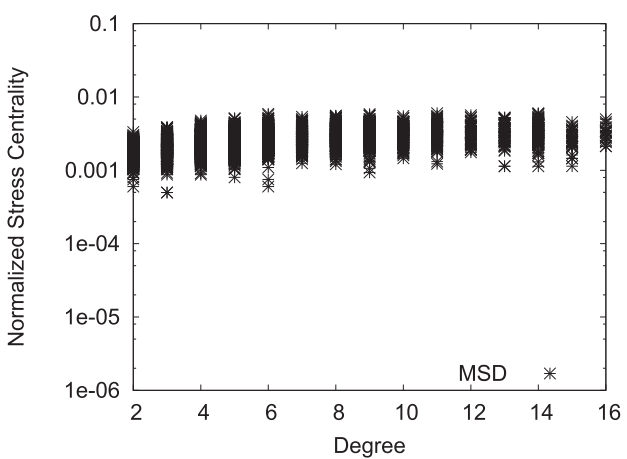

Fig. 9 Normalized SC of each link (MSD, Waxman model).

represents a node degree. Thus, each point in the graphs indicates a normalized SC on a link attached to a node with degree $x$.

First, if $p$ is set to 0.20 , which is relatively close to 0 , the relationship between the normalized SC of each link and the node degree of each link in the $p$-norm based routing metric is similar to that in both the MMD and the MSD schemes, as shown in Fig. 10. When $p$ approaches 0, since the value of $x_{i}^{p}$ ( $x_{i}$ is a node degree) is close to 1 , the number of hops on a route from a source to its destination is dominant in the $p$-norm of the node degree vector. Thus, the transmission route in the $p$-norm routing metric with the small $p$ almost the same as the shortest path.

In contrast, as shown in Fig. 12, if $p$ increases and approaches 1.0, the normalized SC of a link attached to high- 


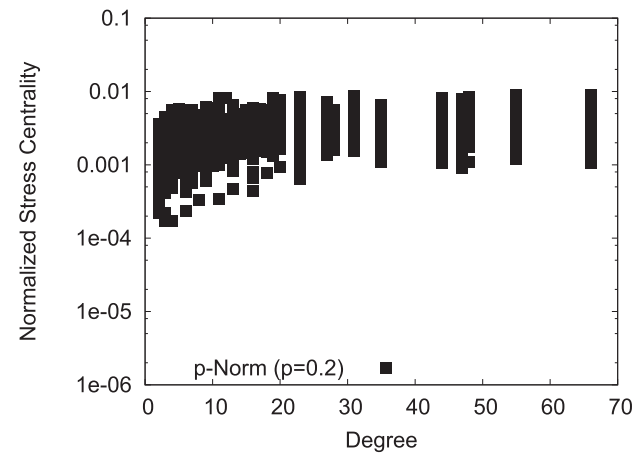

Fig. 10 Normalized SC of each link (Proposed scheme $(p=0.2)$, BA model).

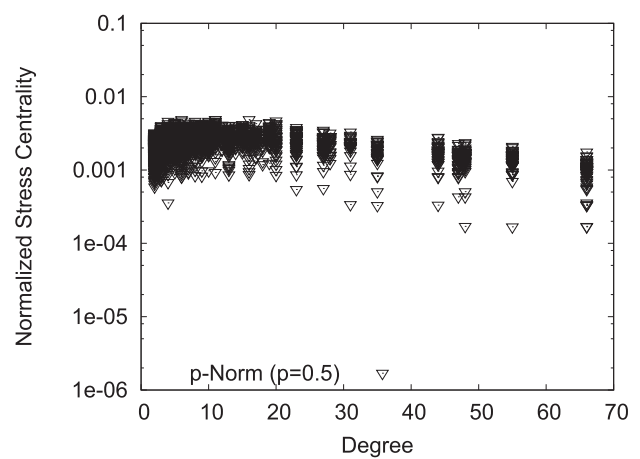

Fig. 11 Normalized SC of each link (Proposed scheme $(p=0.5)$, BA model).

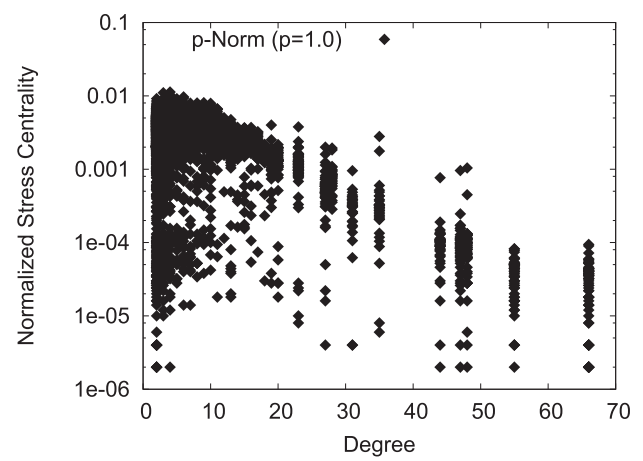

Fig. 12 Normalized SC of each link (Proposed scheme ( $p=1.0$ ), BA model).

degree nodes is drastically reduced, while that attached to low-degree nodes slightly increases. Furthermore, the difference between the normalized SC of each link at highdegree nodes and that at low-degree nodes becomes large as $p$ increases. When $p=1.0$, a route minimizing the sum of the node degrees is selected as a forwarding route. This is because the sum of the node degrees along a path is dominant in the $p$-norm of the node degree vector if $p$ is close to 1.0. Note that, the route is different from the route selection in the MSD scheme, because the forwarding route is one of the shortest paths in the MSD scheme. In the proposed scheme with $p=1.0$, a route having a minimum sum

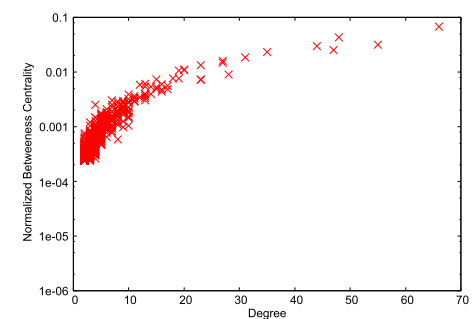

Fig. 13 Normalized BC of each link (Common SPF, BA model).

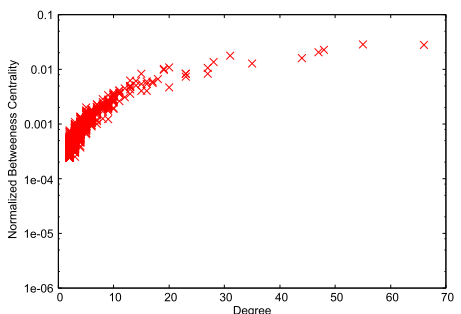

Fig. 14 Normalized BC of each link (MMD, BA model).

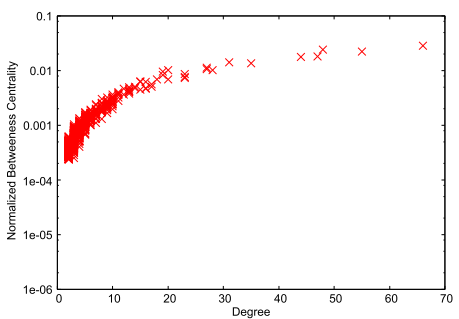

Fig. 15 Normalized BC of each link (MSD, BA model).

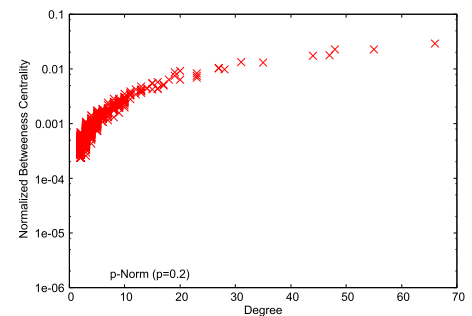

Fig. 16 Normalized BC of each link (Proposed scheme ( $p=0.2)$, BA model).

of node degrees is selected, and no constraint is placed on the number of hops of the route.

Therefore, we conclude that a routing metric based on the $p$-norm of the node degree vector can control the selected route's characteristics by the parameter $p$. If $p$ is relatively small and approaches 0 , then the hop count is dominant for selecting a transmission route. In contrast, if $p$ is relatively large and approaches 1.0, then the sum of the node degrees of a route is dominant rather than the hop count of a route.

In addition, we show the characteristics of $\mathrm{BC}$ in Figs. 13 to 18 for reference because $\mathrm{BC}$ is also used for parameter for evaluation. We cannot see from the figures that 


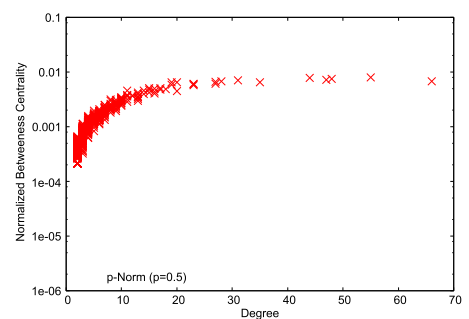

Fig. 17 Normalized BC of each link (Proposed scheme $(p=0.5)$, BA model).

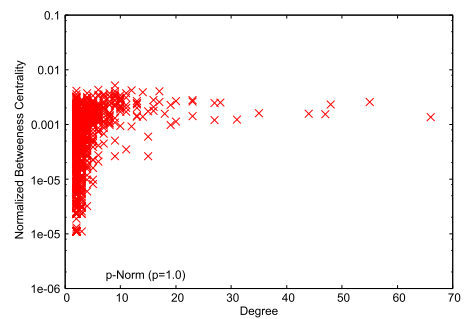

Fig. 18 Normalized BC of each link (Proposed scheme $(p=1.0)$, BA model).

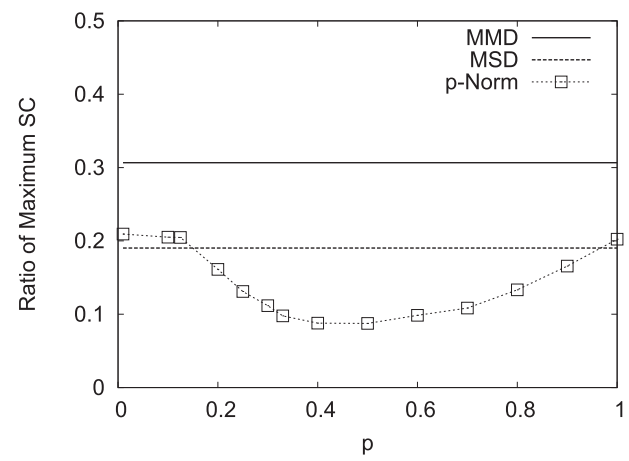

Fig. 19 BA topology (Maximum normalized SC vs. $p$ ).

$\mathrm{BC}$ and SC have very different characteristics.

\subsection{Impact of $p$}

We investigate the impact of $p$ on the normalized SC in the BA network. Figure 19 shows the ratio of the maximum normalized SCs among all links of the MMD, the MSD, and the proposed scheme to those of the common SPF routing in the BA network, and Fig. 20 shows the ratio of the coefficient of variation (CV) of the normalized SC of all links of the $\mathrm{MMD}$, the MSD, and the proposed scheme to those of the common SPF routing. Hence, if the $y$-axis is equal to 1.0, then the value of the maximum normalized $\mathrm{SC}$ or the value of the $\mathrm{CV}$ of the normalized $\mathrm{SC}$ of each scheme is equal to that of the common SPF routing, and if the $y$-axis is less than 1.0 , then those values are less than that of the common SPF routing.

As shown in the figures, we can see the appropriate value of $p$ for minimizing the maximum normalized $\mathrm{SC}$ and the $\mathrm{CV}$ of the normalized SC in the proposed scheme. Fur-

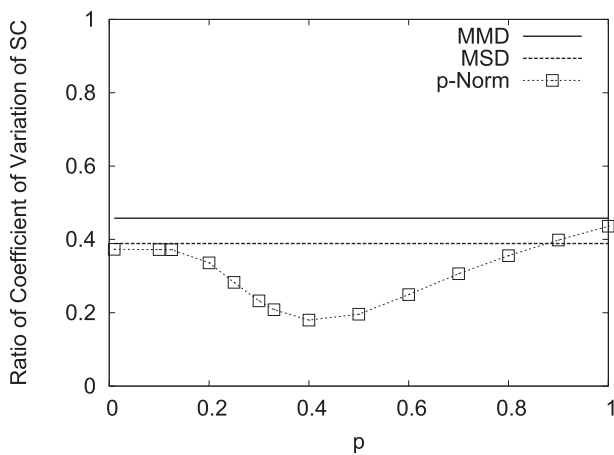

Fig. 20 BA topology (CV of normalized SC vs. $p)$.

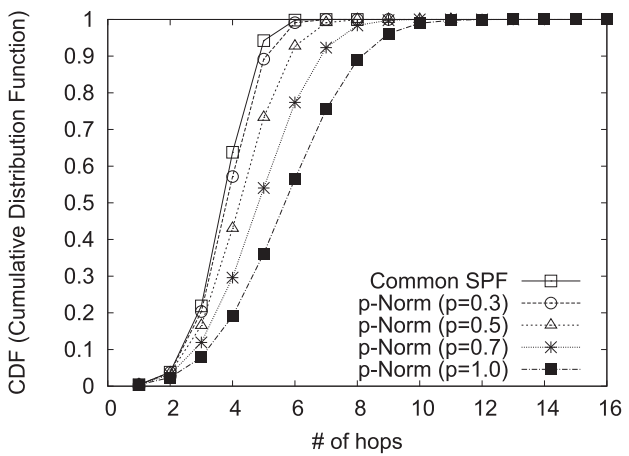

Fig. 21 Cumulative distribution function of all routes in the network (Proposed scheme, BA model).

thermore, both the maximum normalized SC and the CV of the normalized SC of the proposed scheme are minimized compared to those of the other schemes. In the BA network, the maximum normalized SC of the proposed scheme drastically decreases to approximately $10 \%$ of that of the common SPF routing when $p=0.5$. In addition, the CV of the normalized SC is minimized when $p=0.4$, and then the $\mathrm{CV}$ of the proposed scheme decreases to approximately $20 \%$ of that of the common SPF routing. Thus, the proposed scheme is the most effective scheme for avoiding the traffic concentration in a particular link in the BA network.

Furthermore, in the proposed scheme, both the maximum normalized SC and the CV of the normalized SC strongly depend on $p$. Therefore, we can control the effect of the traffic distribution by the parameter $p$. The reason for controlling the traffic distribution effect by $p$ is that the number of hops of each route can be controlled by $p$. Figure 21 shows the Cumulative Distribution Function (CDF) of the number of hops of a route in the BA network. As shown in Fig. 21, we can see that the CDF of the number of hops in the proposed scheme is almost the same as that in the common SPF routing if $p$ is approximately equal to 0 . Then, the maximum SC becomes large because the number of hops for data transmission between a source node and its destination node are minimized by using the shortest path, and because some links of the network are highly used by the shortest path. In contrast, the number of hops of each route increases as $p$ increases. This means that the number 


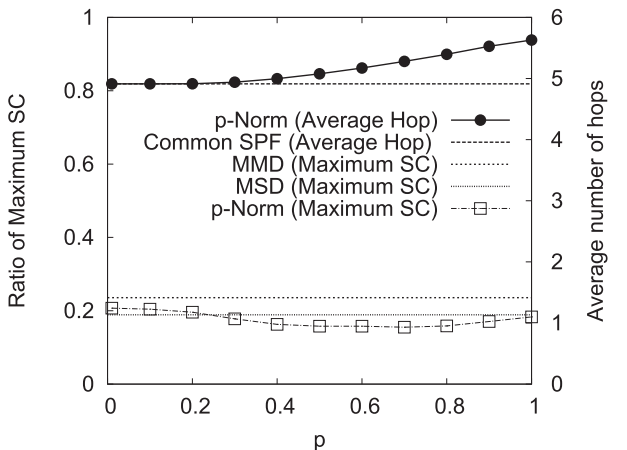

Fig. 22 Waxman model.

of hops between a source node and its destination node is larger than that in the common SPF routing. However, links used for data transmission are not concentrated on particular links of the networks and are distributed as the number of hops of a route increases. Whereas, when $p$ becomes too large, links used for data transmission tends to be concentrated on particular links in the networks more and more, because the number of hops of each route increases. As a result, the maximum SC of the network increases if $p$ becomes too large. Therefore, the maximum normalized SC and the $\mathrm{CV}$ of the normalized $\mathrm{SC}$ are minimized by a certain value of $p$. In the BA network, the optimal value of $p$ minimizing the maximum $\mathrm{SC}$ of the network and the $\mathrm{CV}$ of the normalized SC is 0.4 as shown in Fig. 19 and 20.

As discussed above, we can conclude that the route longer than the shortest one is effective for minimizing the maximum SC in the network. Then, the routing metric calculated by both the node degree along a route and the definition of the $p$-norm is effective in deciding a route other than the shortest path.

\subsection{Impact of Network Topology}

In the previous subsection, we investigate the performance of our proposed scheme in the network modeled by the BA model. Here we investigate the applicability of the proposed scheme in other network topologies. In this subsection, we use the Waxman network topology and the three router-level topologies of real ISP networks shown in Table 1; Figures 26, 27 and 28 illustrate how many nodes are equipped with some, say $k$, links in the three router-level topologies.

Figures 22, 23, 24, and 25 show the ratio of the maximum normalized SC of the MMD, MSD, and the proposed scheme to that of the common SPF routing. We focus only on the maximum normalized SC, because the tendency of the $\mathrm{CV}$ of the normalized SC is similar to the maximum normalized SC. As shown in the figures, we can see that the proposed scheme is the most effective for reducing the maximum SC. In addition, an optimal value of $p$ minimizing the maximum SC is seen in each topology. Then, the optimal $p$ minimizing the maximum SC differs in each topology. However, the optimal value of $p$ in each topology

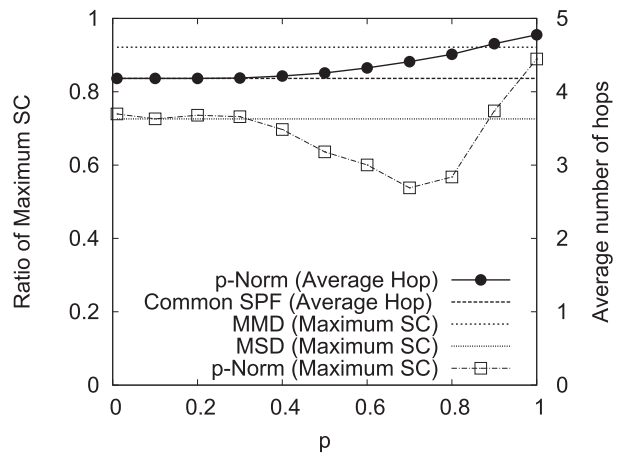

Fig. 23 Sprint network.

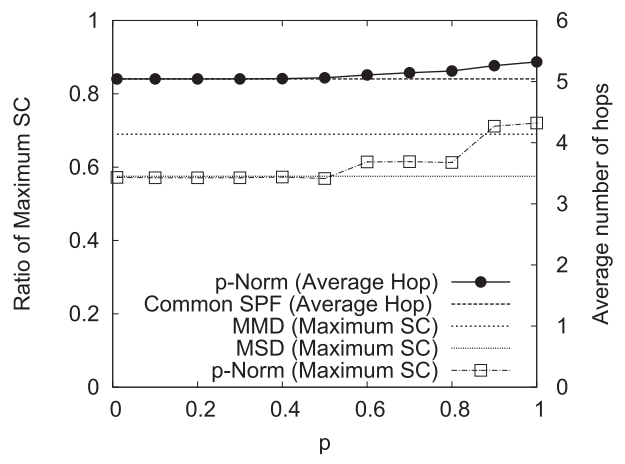

Fig. 24 AT\&T network.

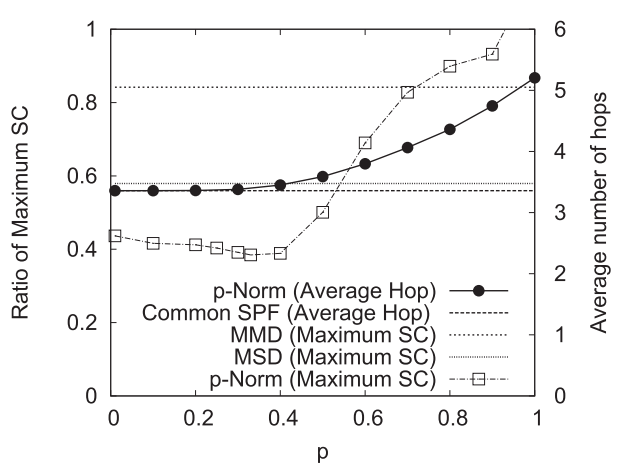

Fig. 25 Level3 network.

ranges from 0.4 to 0.7 . The effectiveness of the proposed scheme with the optimal $p$ is that the maximum normalized SC is reduced to $15 \%$ of that of the common SPF routing in the Waxman model. Furthermore, it is approximately a $50 \%$ reduction in the Sprint network, approximately a $60 \%$ reduction in the AT\&T network, and approximately a $40 \%$ reduction in the Level 3 network.

In addition, we investigate the increment of the number of hops of each route in the proposed scheme to estimate the impact of the packet forwarding performance of each data transmission. Figures 22, 23, 24, and 25 also show the comparison of the average number of hops in each route as a function of $p$ between the common SPF routing and the proposed scheme. In each topology, the increment of the average number of hops of the proposed scheme compared 


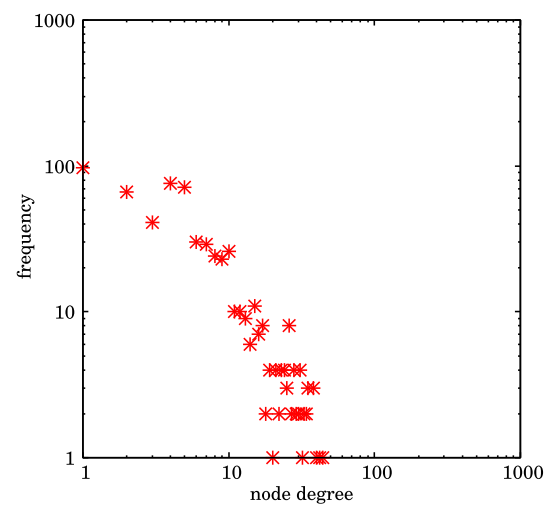

Fig. 26 Sprint network.

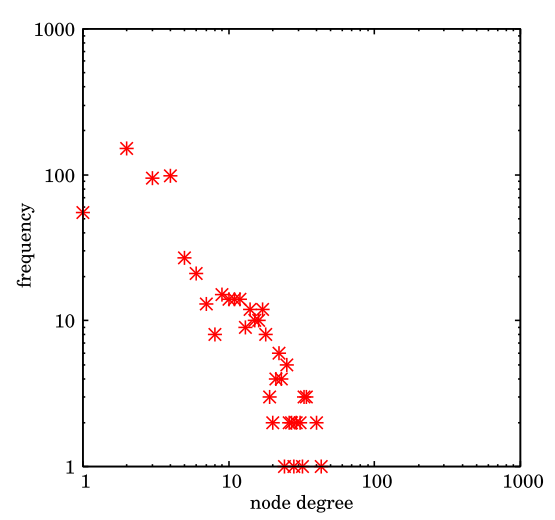

Fig. 27 AT\&T network.

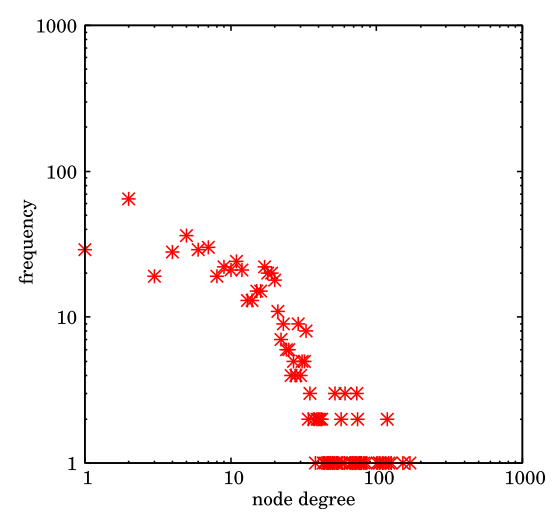

Fig. 28 Level3 network.

to the common SPF routing is more than 0 and less than 1 at the optimal $p$ minimizing the maximum SC. Therefore, no significant impact on packet forwarding performance (i.e., transmission delay) is found when the proposed scheme is applied.

In the Level3 Network (AS3356), there are many paths from source to destination because average of node degree is high and many nodes have high number of degree in Fig. 28, and the maximum SC is well reduced when $p$ is close to 0 ; i.e., relatively, node degree does not play an important role. In Sprint Network (AS1239), the path selection is limited because the average of node degree is low and many of the node degree is less than 10 in Fig. 27, there are not many paths from source to destination because the average node degree is low. The node degree plays an important role here in traffic load distribution; the maximum SC is minimized when $p$ is around 0.7. On the other hand, in AT\&T Network (AS7018), the number of paths from source to destination is quite limited because many nodes are with low degree such as one to four as shown in Fig. 27, and the maximum SC does not heavily depend on $p$, whereas that is better than with SPF.

Thus, we can conclude that our proposed scheme is effective in each network topology and can also be adapted to real ISP topologies by controlling $p$. In addition, we can see that the optimal $p$ of each topology takes values ranging from 0.4 to 0.7 in our topology models. Note that, we need to set the optimal $p$ for minimizing the maximum SC; however, finding the optimal $p$ is future work and beyond the scope of this paper. However, we saw that the $p$ takes values of 0.4 or 0.5 , if the proposed scheme achieves the optimal or the sub-optimal performance in our topology models. Therefore, if the proposed scheme is applied to other network topologies, $p$ is set to 0.4 or 0.5 .

\section{Conclusions}

This paper provided a definition of node-to-node distance in networks based on both the definition of the norm and the node degree. In addition, we explained how to apply the node-to-node distance to the OSPF routing protocol. Through simulation results, we clarified the performance of the proposed scheme in some network topologies including real ISP topologies, by the maximum SC and the coefficient of variation of the SC as performance measures of the congestion possibility of a network link.

The effectiveness of our proposed scheme is that it can control the number of hops of the transmission path by the parameter $p$ of the norm; that is, a small $p$ makes the route selection with the shortest number of hops, while a large $p$ makes the route selection based on the node degree as well as the number of hops. Furthermore, we clarified that a route slightly longer than the shortest one is effective for minimizing the maximum SC in networks. The optimal $p$ of our proposed scheme makes the transmission route longer than the shortest path. However, the increment of the number of hops is less than 1 hop. Hence, the transmission performance is not degraded by applying the proposed scheme.

Furthermore, we saw that the parameter $p$ should set an optimal value ranging from 0.4 to 0.7 depending on the network topology to minimize the maximum SC and the coefficient of variation of the SC. Lastly, the detailed relationship between the network topology and its appropriate $p$ will be an interesting research topic in future work.

\section{Acknowledgements}

This work was supported in part by the Japan Society for the 
Promotion of Science, Grant-in-Aid for Scientific Research (C) (No.23500090), and Electronics Research Laboratory, Fukuoka Institute of Technology.

\section{References}

[1] D. Awduche, A. Chiu, A. Elwalid, I. Widjaja, and X. Xiao, "Overview and principles of Internet traffic engineering," RFC3272, May 2002.

[2] B. Fortz and M. Thorup, "Internet traffic engineering by optimizing OSPF weights," Proc. IEEE Infocom2000, pp.519-528, March 2000.

[3] M. Faloutsos, P. Faloursos, and C. Faloutsos, "On power-law relationships of the Internet topology," ACM SIGCOMM CCR, vol.29, Issue 4, pp.251-262, Oct. 1999.

[4] P. Mahadevan, D. Krioukov, M. Fomenkov, B. Huffaker, X. Dimitropoulos, KC Claffy, and A. Vahdat, "The Internet AS-level topology: Three data sources and one definitive metric," ACM SIGCOMM CCR, vol.36, Issue 1, pp.17-26, Jan. 2006.

[5] CAIDA, "skitter - CAIDA's Router-Level Topology Measurements," http://www.caida.org/tools/measurement/skitter/ router_topology/index.xml

[6] L. Adamic and B. Huberman, "Zipf's law and the Internet," Glottometrics, vol.3, no.1, pp.143-150, 2002.

[7] S. Lim, C. Yu, and C.R. Das, "A realistic mobility model for wireless networks of scale-free node connectivity," Int. J. Mobile Commun., vol.8, Issue 3, May 2010.

[8] N. Spring, R. Mahajan, D. Wetherall, and T. Anderson, "Measuring ISP topologies with rocketfuel," IEEE/ACM Trans. Netw., vol.12, no.1, pp.2-16, Feb. 2004.

[9] D. Alderson, L. Li, W. Willinger, and J.C. Doyle, "Understanding Internet topology: Principles, models, and validation," IEEE/ACM Trans. Netw., vol.13, no.6, pp.1205-1218, Dec. 2005.

[10] P. Mahadevan, D. Krioukov, K. Fall, and A. Vahdat, "Systematic topology analysis and generation using degree correlations," Proc. ACM SIGCOMM 2006, pp.135-146, Sept. 2006.

[11] H. Haddadi, S. Uhlig, A. Moore, R. Mortier, and M. Rio, "Modeling Internet topology dynamics," ACM Comput. Commun. Rev., vol.38, no.2, pp.65-68, April 2008.

[12] G. Yan, T. Zhou, B. Hu, Z.Q. Fu, and B.H. Wang, "Efficient routing on complex networks," Phys. Rev. E 73, vol.73, no.4, 046108, April 2006.

[13] Z.X. Wu, G. Peng, Eric W.M. Wong, and K.H. Yeung, "Improved routing strategies for data traffic in scale-free networks," J. Statistical Mechanics: Theory and Experiment, no.11, P11002, Nov. 2008.

[14] J. Shimada, N. Natsui, H. Tamura, M. Uchida, T. Ikenaga, and Y. Oie, "Path selection scheme for traffic load distribution considering the characteristics of network topology," ISCE 2009, pp.876-880, May 2009.

[15] A.-L. Barabási and R. Albert, "Emergence of scaling in random networks," Science, vol.286, no.5439, pp.509-512, Oct. 1999.

[16] A. Shimbel, "Structural parameters of communication networks," Bulletin of Mathematical Biophysics, vol.14, no.4, pp.501-507, Dec. 1953.

[17] "Rocketfuel: An ISP topology mapping engine," http://www.cs.washington.edu/research/networking/rocketfuel/

[18] H. Tamura, M. Köppen, M. Uchida, M. Tsuru, and Y. Oie, "Node degree-aware link cost for traffic load-distribution in large-scale networks," INCOS, pp.299-304, 2011 Third International Conference on Intelligent Networking and Collaborative Systems, 2011.

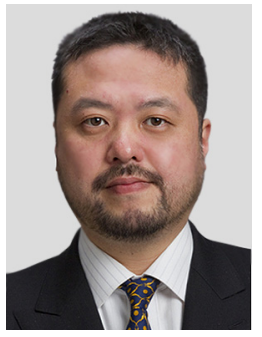

Jun'ichi Shimada receieved the B.E. and M.E. degrees from Kyoto University, Kyoto, Japan in 1991 and 1993, respectively. After receiving M.E. degree, he worked at the Ministry of Posts and Telecommunications (1993-1996), the Ministry of Foreign Affairs of Japan (19961998), the Ministry of Posts and Telecommunications (1998-2000), the Ministry of Public Management, Home Affairs, Posts and Telecommunications (2000-2002), the Communications Research Laboratory (2002-2004), the National Institute of Information and Communications Technology (20042006), and the Ministry of Internal Affairs and Communications (20062011). Since 2011, he has been a Research Professor in the Dependable Network Innovation Center, Japan Advanced Institute of Science and Technology. His research interests include ICT policy and network architecture.

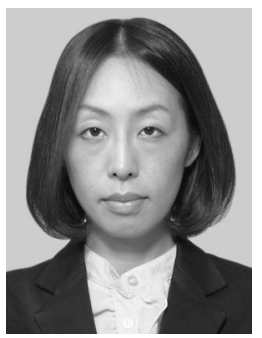

Hitomi Tamura received the B.E., M.E., and D.E. degrees in Computer Sciences and Electronics from Kyushu Institute of Technology, Iizuka, Japan in 2000, 2002 and 2005, respectively. From 2005 to 2007, she was a PostDoctoral Researcher in the Department of Computer Science and Systems Engineering, Kyushu Institute of Technology. From 2007 to 2008, she was a Researcher in the Human Media Creation Center/Kyushu. From 2008 to 2011, she was an Assistant Professor in the Network Design Research Center, Kyushu Institute of Technology. Since 2011, she has been an Assistant Professor in the Faculty of Engineering, Fukuoka Institute of Technology. Her research interests include computer networks. She is a member of the IEEE and ACM.

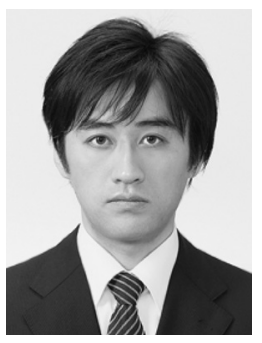

Masato Uchida received B.E., M.E., and D.E. degrees from Hokkaido University, Hokkaido, Japan, in 1999, 2001, and 2005, respectively. In 2001, he joined NTT Service Integration Laboratories, Tokyo, Japan. From August 2005 to March 2012, he was an Associate Professor in the Network Design Research Center, Kyushu Institute of Technology, Fukuoka, Japan. Since April 2012, he has been an Associate Professor in the Department of Electrical, Electronics and Computer Engineering, Faculty of Engineering, Chiba Institute of Technology, Chiba, Japan. His research field is mathematical information engineering with applications to information networking and machine learning. He is a member of the IEEE and ACM.

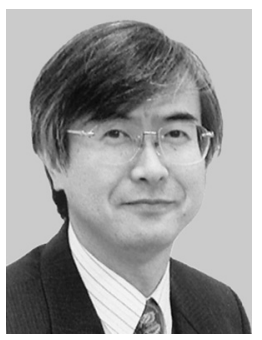

Yuji Oie received B.E., M.E. and D.E. degrees from Kyoto University, Kyoto, Japan in 1978, 1980 and 1987, respectively. From 1980 to 1983 , he worked at Nippon Denso Company Ltd., Kariya. From 1983 to 1990, he was with the Department of Electrical Engineering, Sasebo College of Technology, Sasebo. From 1990 to 1995 , he was an Associate Professor in the Department of Computer Science and Electronics, Faculty of Computer Science and Systems Engineering, Kyushu Institute of Technology, Iizuka. From 1995 to 1997, he was a Professor in the Information Technology Center, Nara Institute of Science and Technology. Since April 1997, he has been a Professor in the Department of Computer Science and Electronics, Faculty of Computer Science and Systems Engineering, Kyushu Institute of Technology. His research interests include performance evaluation of computer communication networks, high speed networks, and queueing systems. He is a fellow of the IPSJ and a member of the IEEE. 